\title{
Requirements Analysis of Art Teachers for Robot-Aided Classes
}

\author{
Eun-Mi Lee and Kang-Hee Lee \\ Department of Digital Media, Soongsil University, \\ 369 Sangdo-Ro, Dongjak-Gu, Seoul, 06978, South Korea \\ kanghee.lee@ssu.ac.kr
}

\begin{abstract}
In South Korea, the government has tried to deploy robotic systems as teaching aids in elementary and middle schools. However, the practical use of teaching assistant robots in classrooms has remained limited since teachers have experienced difficulties in using the legacy robot scenario editor to design their own class scenario. To provide flexible and on-demand class scenarios, teachers should edit class scenarios that are designed to achieve their class objectives. Since most teachers are usually not familiar with computer programming and robotics, it is important to have a class scenario editor that is easy to use without much needed expertise. We analyze the requirements for developing a class scenario editor for robot-aided classes by administering a questionnaire and conducting interviews with art teachers. From the user requirements, a class scenario editor for robot-aided classes can be developed and used by typical teachers without needing specialized expertise.
\end{abstract}

Keywords: robot-aided class; class scenario editor; teaching assistant robot

\section{Introduction}

Using educational robot-based learning systems in classrooms has been demonstrated to afford a significant advantage for students by improving overall learning interest and motivation [1-3]. In South Korea, the government has tried to deploy robotic systems as teaching aids in elementary and middle schools to utilize the effect of robot-aided classes. The government, teachers, and robot companies have tried to achieve efficient and feasible robot-aided classes. However, regardless of experiments in model schools over several years, the practical use of teaching assistant robots in the classroom has remained very limited in Korea since teachers have experienced difficulties in using the legacy robot scenario editor to design their own class scenario. Most teachers merely play limited class scenarios based on the example template, and students usually lose interest in robotaided classes. To provide flexible and on-demand class scenarios, teachers should be able to edit class scenarios that are designed to achieve their class objectives.

Software tools and scripting languages for robot service scenario edits have been developed for the development of robot applications and service scenario [4-7]. These tools and languages provide various ways to design robot navigation scenarios, robot motion scenarios, and robot service scenarios. However, most existing tools and languages are only readily accessible to experts of computer programming or robotics. Recently, the expansion of the market for service robots has led to the development of the service scenario editor for ordinary users. In [5], a robot scenario script language and tool for teachers who are non-experts was proposed, but its use remains difficult for teachers due to a lack of understanding of user requirements. Most teachers, except a few with computer programming expertise, failed to utilize the robot scenario editor. Finally, they took the editor software back and requested a modified version that can be used by typical teachers. 
Understanding user requirements is an integral part of information systems design and is critical to the success of interactive systems. It is now widely understood that successful systems and products begin with an understanding of the needs and requirements of the users. As specified in the ISO 13407 standard (ISO, 1999), usercentered design begins with a thorough understanding of the needs and requirements of the users. The benefits can include increased productivity, enhanced quality of work, reductions in support and training costs, and improved user satisfaction [8].

In this paper, we analyze the requirements for developing the class scenario editor for robot-aided classes by administering a questionnaire and conducting interviews with teachers. From the user requirements analysis, we define the user requirements to design a class scenario editor for robot-aided classes that can be used by typical teachers without expertise about computer programming and robotics. With the fast prototyping method, the defined user requirement will be verified and enhanced.

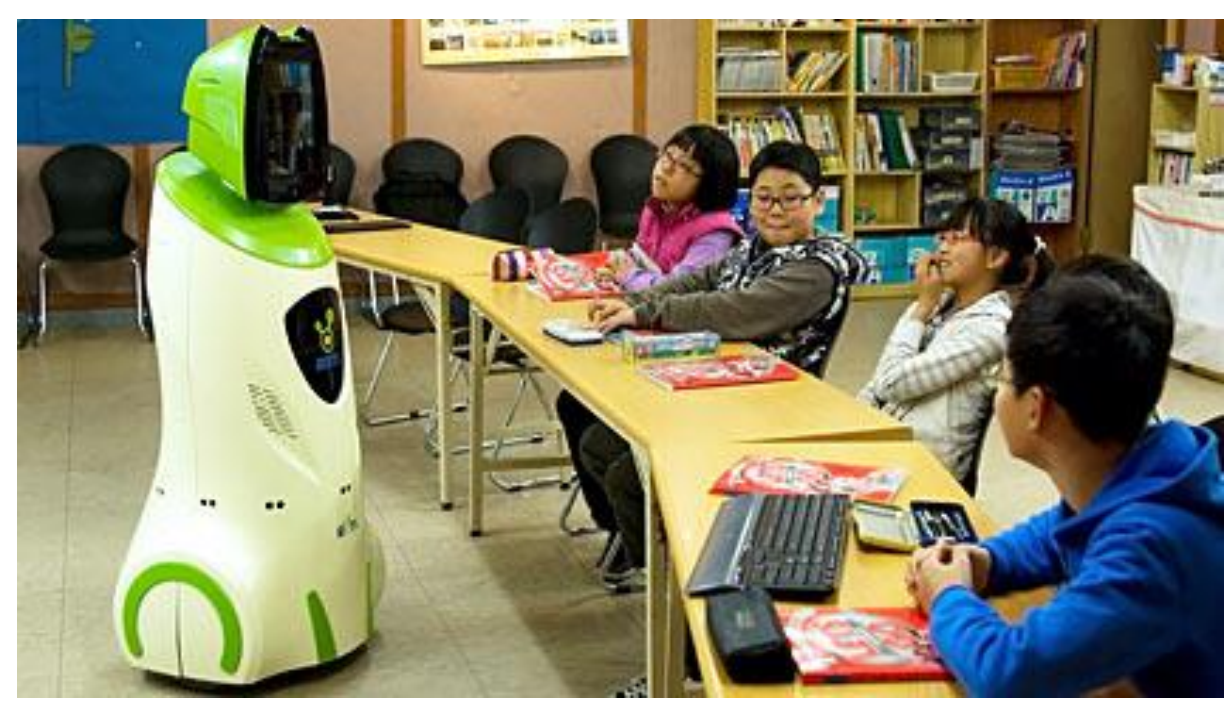

Figure 1. Robot In An English Class In Korea

\section{Related Works}

In this section, we review the existing robot scenario editors, and thus lay the foundation for the present study purpose.

\subsection{Robot Scenario Editors}

Service robots for personal and domestic use are becoming increasingly common nowadays. Easy and efficient programming of such robots is an enormous research and commercial space that is also beginning to be explored [9]. As shown in Figure 2, legacy robot scenario editors provide functions and interfaces for the engineers and programmers who design the user scenario. Editors create the robot scenario with the scenario editor in the PC, which then translates the scenario into the scenario script. The robot loads the scenario script and the script engine in the robot performs the specific service based on the scenario and user command, including controlling remote controller, sound to text (STT), input through mobile app and so on.

In [6], a robot scenario editor was proposed for use by typical teachers. The robot scenario editor is composed of two modules: a robot scenario editor that creates a scenario script and a robot scenario interpreter to run the script. The script editor consists of Import, Package, and Scenario. The scenario interpreter module is responsible for 
execution of scenario by analyzing the scenario script file that has been created in the scenario editor. The interpreter can be performed with the analysis of the script, and control multimedia playback.

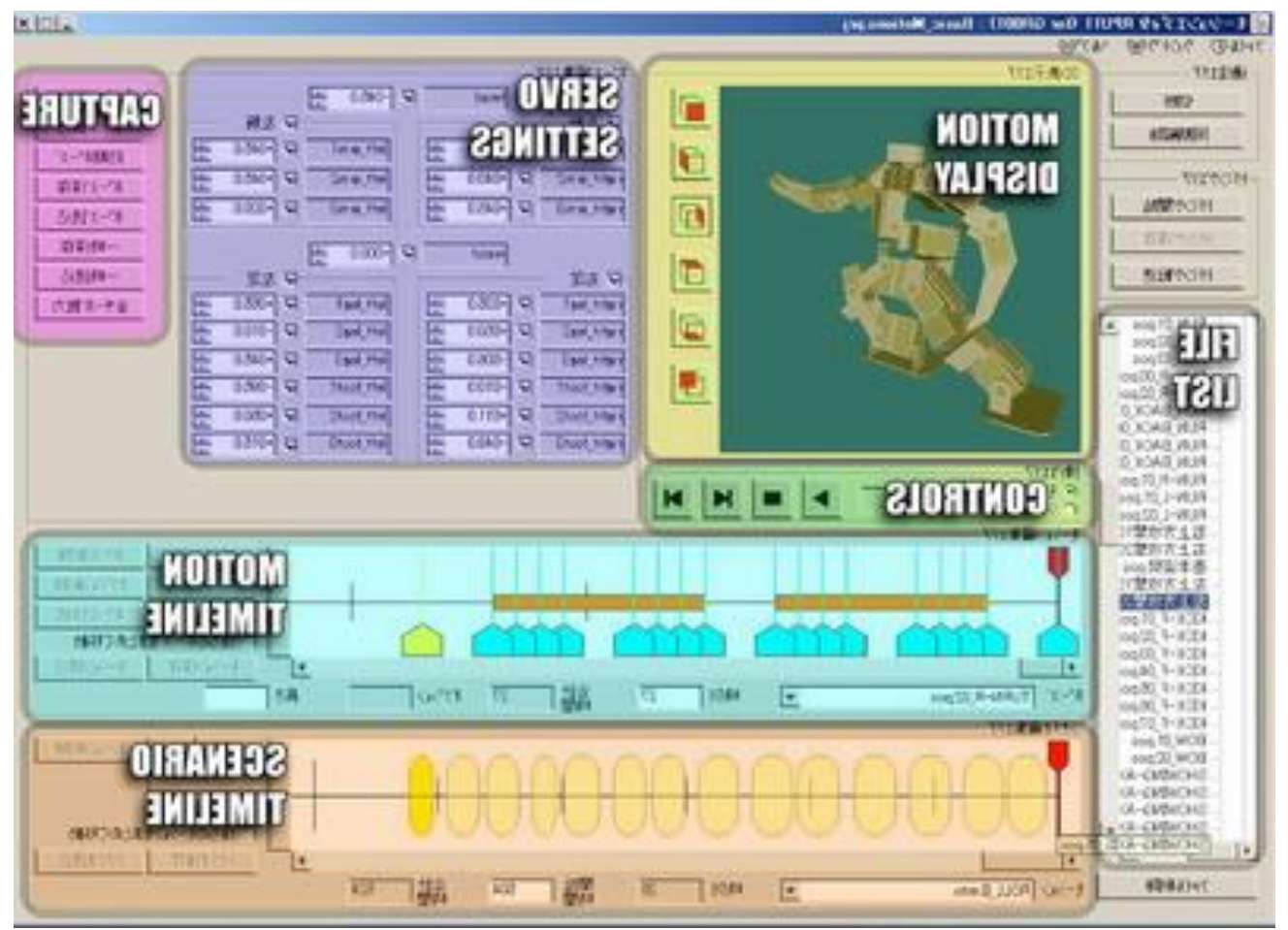

Figure 2. Example of Robot Scenario Editor [13]

The robot scenario editor proposed by [6] is much more user-friendly than existing robot scenario editors. However, it remains too difficult to be used by typical teachers. In the beta test, among ten teachers, only one teacher could use the scenario editor with limited functions, and the remaining nine teachers could barely do anything although they had been given an hour lesson from the developer and manuals. Anthropomorphized robots are regarded as beneficial tools in education due to their capabilities for improving teaching effectiveness and learning motivation [10]. In [10], the script languages and the authoring tool were proposed for teachers to design an English as a foreign language (EFL) class. However, according to the teacher's opinions in [10], teachers without professional computer experience would have to spend more time learning the authoring tool to design the robot's movements.

\subsection{Motivation}

The developers in [6] concluded that they failed to define the user requirements of teachers for robot scenario editors. To modify and enhance their editor, they decide to analyze the user requirements. Requirements analysis in systems engineering and software engineering encompasses those tasks that go into determining the needs or conditions that must be met for a new or altered product or project, taking account of the possibly conflicting requirements of the various stakeholders, analyzing, documenting, validating and managing software or system requirements [11]. In [10], the authors try to overcome the difficulties that teachers have in trying to learn the authoring tool by providing more motion example. We have to provide the class scenario editor to apply all subjects in schools and various class objectives of teachers. In [12], we proposed a class scenario design method, however, it was limited to art class. 
Stakeholder interviews are a common technique used in requirements analysis. In our case, we assume our stakeholders are typical teachers who have no expertise of computer programming and robotics. The interview can serve as a means of obtaining highly focused information. Moreover, the personal nature of the interviews provides a more relaxed environment and derives a deeper understanding about the user requirements.

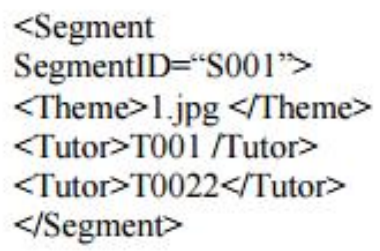

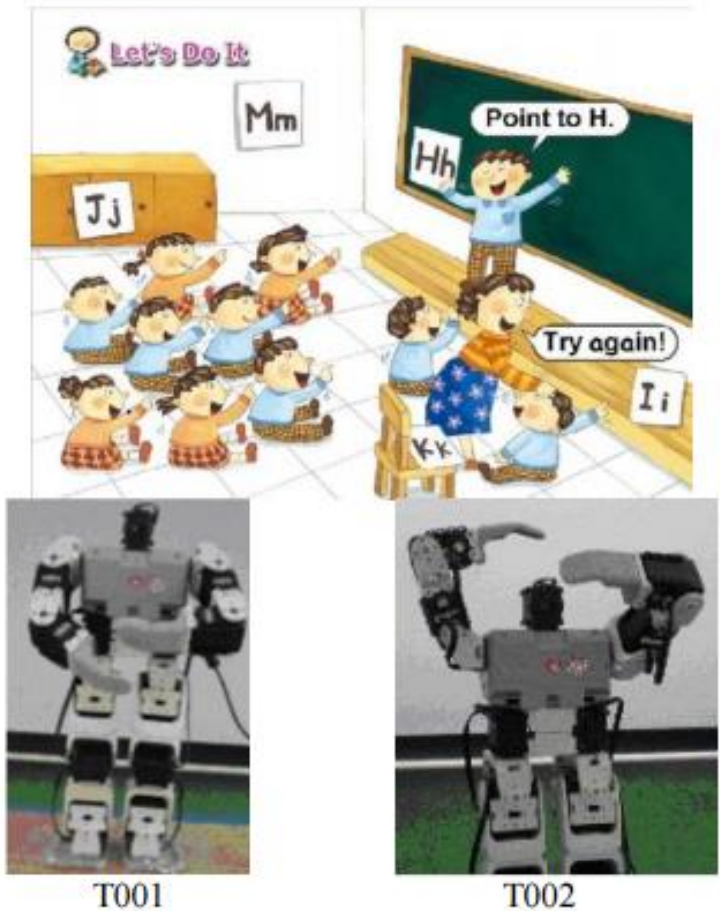

Figure 3. Segment Script in [11]

\section{Requirements Analysis by Interview}

To analyze the user requirements for the class scenario editor, we interviewed ten teachers who had used the legacy class scenario editor in model schools. We requested them to answer the questionnaire, as shown in Table 1, to gather basic information for the interview. Based on the questionnaire result, we conducted close one-to-one interviews to analyze the user requirements of the class scenario editor for robot-aided classes.

We adapted questions in the interview to obtain detailed information of individual teacher's requirements according to the answers in the questionnaire. For example, if a teacher answered strongly positive to Q5, we made detailed questions about the motion editor. The class scenario editor provides two kinds of motion editor. The first kind is based on Robot plus of Robotis Inc., as shown in Figure 4. Robot plus is a well-designed 3D software solution to edit robot motion by using 3D graphics, robotics mechanics and manual manipulation. However, since teachers usually have no expertise about 3D mechanics, we found that the robot motion editor based on 3D editor is too difficult for teachers to use. The second kind is based on the 3D sensor (Kinect of Microsoft) based motion editor. Since teachers can intuitively edit the robot motion by their own motion, we found the 3D sensor based motion editor to be easier to use for teachers. 
Table 1. Questionnaire for Requirements Analysis

Questionnaire to analyze user requirements of class scenario editor

Below is a list of the ways you might have felt or behaved. Please tell me how often you have felt this way during the past week.

\section{During using class scenario editor}

\begin{tabular}{ccll}
\cline { 2 - 3 } Strongly & Somewhat & Somewhat & Strongly \\
Negative & Negative & Positive & Positive \\
\hline
\end{tabular}

Q1. Are the terms in the class scenario editor easy to understand?

Q 2. Have you used almost all functions in the class scenario editor?

Q 3. Is the robot navigation editing function useful?

Q 4. Is the robot navigation editing function easy to use?

Q 5. Is the robot motion editing function useful?

Q 6. Is the robot navigation editing function easy to use?

Q 7. Have you made a new commend to control the robot?

Q 8. Is the remote controller useful?

Q 9. Is the remote controller mobile app useful?

$\mathrm{Q}$ 10. Is the remote controller based on voice recognition useful?

Q 11. Is the robot helpful to improve the class?

$\mathrm{Q}$ 12. Are there any problems in the robot-aided class?

Q 13. Have you ever created a new class scenario from scratch?

Q 14. Have you ever modified an example template of class scenario?

Q 15. Are the templates of class scenario enough to design your scenario?

Q 16. Do you want to create the templates of class scenario and share to other teachers?

$\mathrm{Q}$ 17. Is the simulator useful to design and review your class scenario?

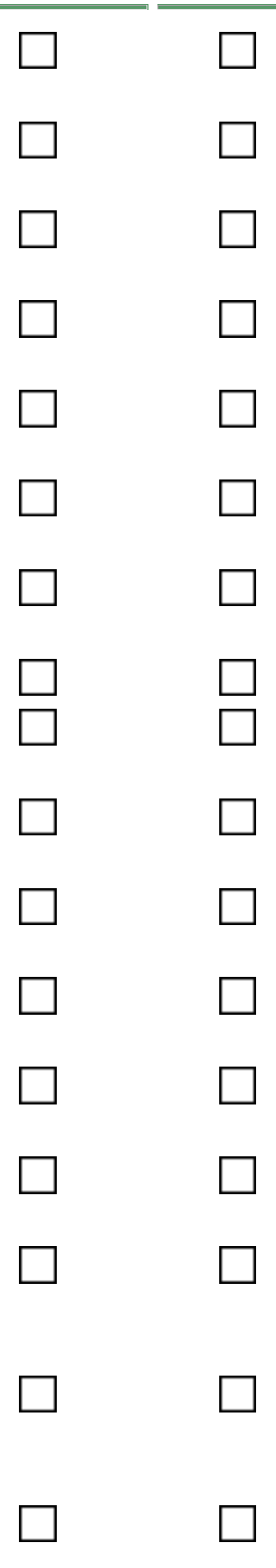

Notice: Any personal information you give to us will be protected. We will use the information only to enhance the class scenario editor and to improve the quality of robot-aided classes. It will also be used to support the research activities for robot-aided classes.

We interviewed teachers about various topics including terms in the editor, navigation editor, motion editor, commander to control the robot, most useful things 
about robot-aided classes, templates, design from scratch, and simulator. An interesting finding was that teachers think the most useful function of the teaching assistant robot is giving a quiz to students. Students usually do not like to have a quiz because they feel evaluated by teachers. However, when a robot gives a quiz to students, they feel it is a form of game and thus is fun to play with robots. Teachers thought that the most powerful function of the robot-aided classes is the possibility to raise the students' interest and to transform a boring class into a funny class. Therefore, the class scenario editor should provide functions capable of designing the class scenario, including the quiz editor to maximize the merit of the robot-aided classes. In addition, we found that the quiz editor should include various levels of quizzes to adapt to the students who have different levels of study achievement. Also, teachers pointed out that the robot gives answers too fast and directly in the example templates, which reduces student interest in quiz time.

Table 2 presents the facts collected from the interviews. We could obtain highly focused knowledge for designing the class scenario editor and a deep understanding about the user requirements.

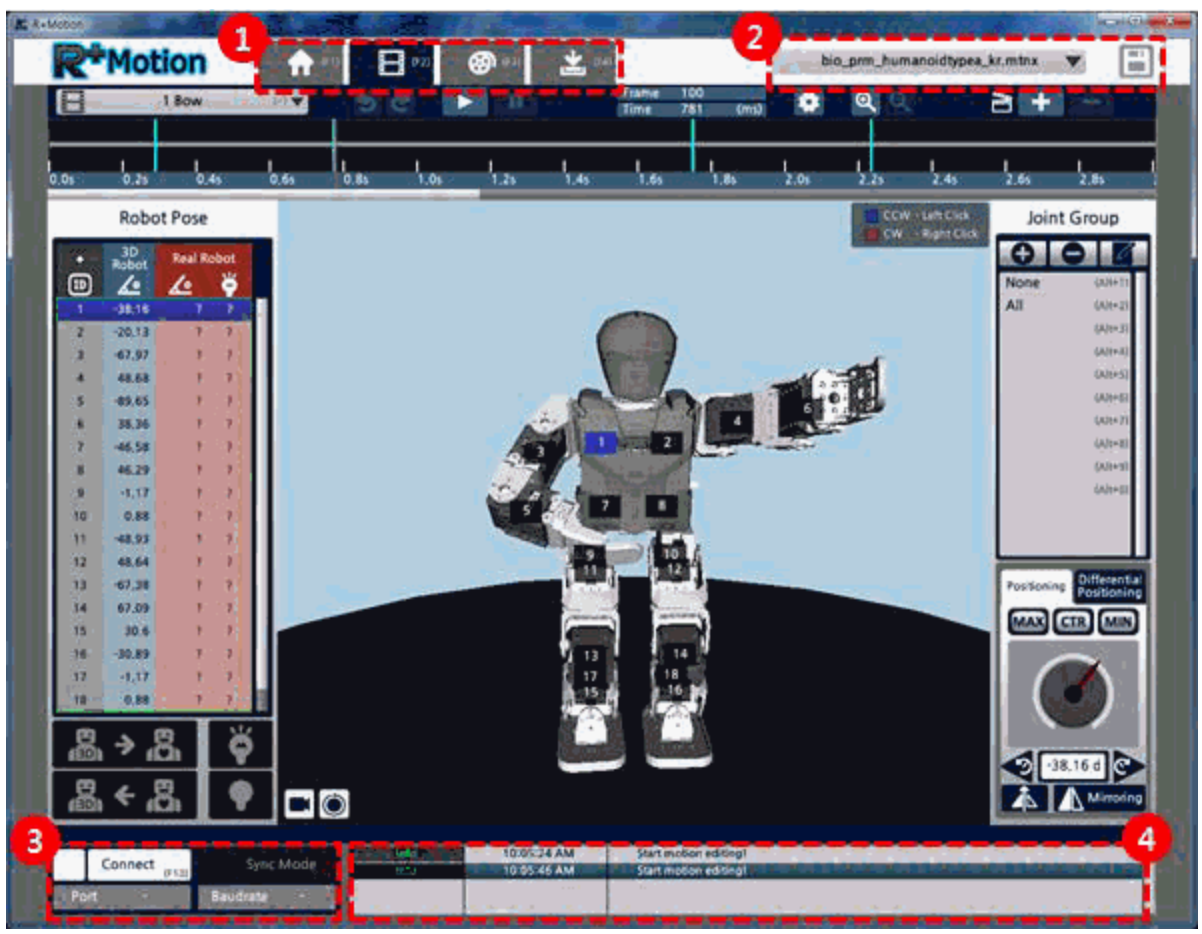

Figure 4. R+Motion of Robotis [14] 


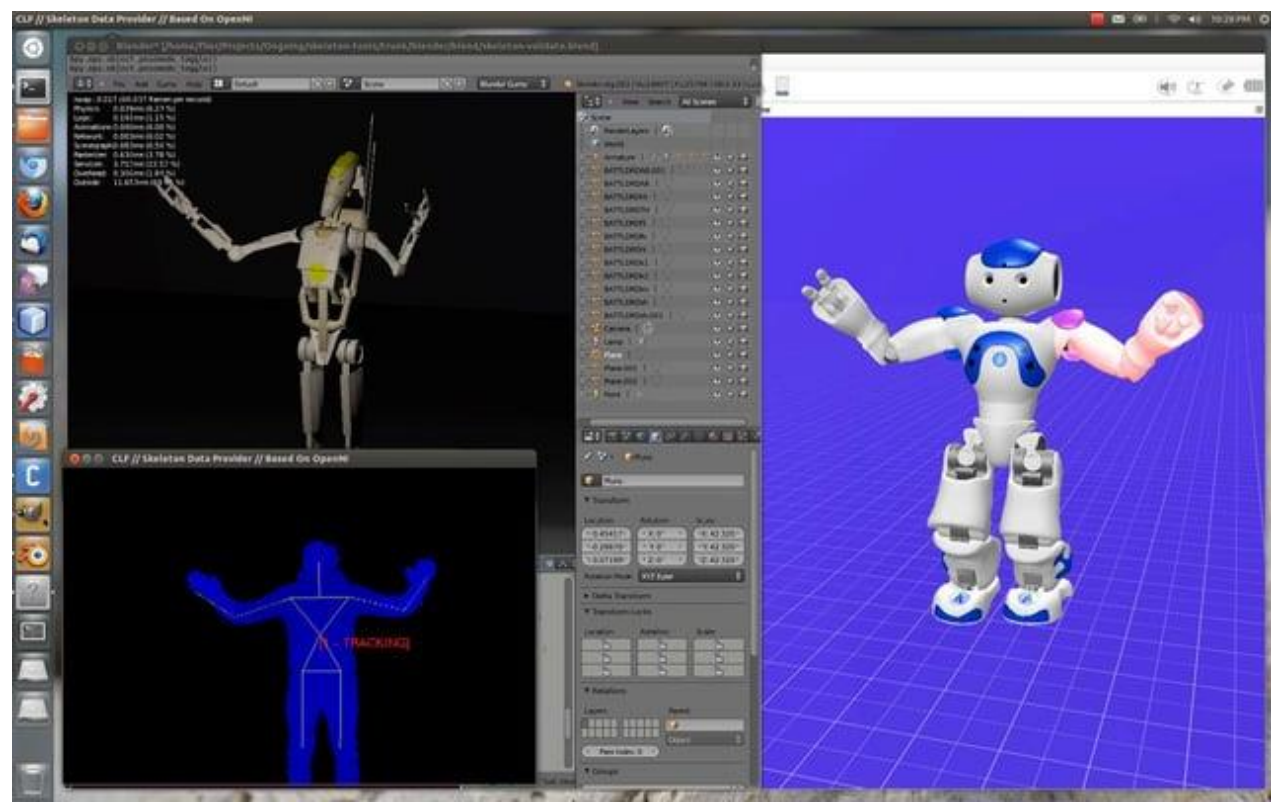

Figure 5. Simultaneous NAO (Choregraphe) and Blender Motion Control via XBox Kinect [15]

Table 2. Facts from the Interviews

\begin{tabular}{|l|c|}
\hline Subject & Facts \\
\hline \hline Terms & Terms in the editor are too difficult. \\
- Terms in the editor are confusing. \\
- Terms of abbreviation are irritating. \\
- Teachers usually have no interest about robot \\
navigation. \\
- Teachers tend to dislike robot movement in the \\
middle of class. \\
- Teachers want to move and gather attention of \\
students just before the beginning of class. \\
- Since the arrangement of desks and chairs often \\
changes, the map for navigation should be easily edited \\
or loaded from saved maps. \\
- Motion editor based on 3D editor is too difficult to \\
be used and time consuming. \\
- Motion editor based on 3D sensors is easy to be \\
used and efficient. \\
- Modifying the motion template is the best way to \\
design a new motion. \\
- Teachers worry about getting hurt by the robot. \\
Students like to be close to robots and robots sometimes \\
move unpredictably. \\
\hline Remote controller is not convenient to use and \\
distracts focusing on teaching. \\
- Controlling by using editor SW is not convenient to \\
use and distracts focusing on teaching. \\
- Controlling by using STT is convenient to use and \\
keeps focusing on teaching. \\
- Students are entertained by mimicking teacher's
\end{tabular}




\begin{tabular}{|l|c|}
\hline Quiz & commanding \\
\hline Teachers love robots to give a quiz to students and \\
think that is the best function of robot-aided classes. \\
- Students feel comfortable and funny when robots \\
give a quiz to them. \\
- Various levels of quiz should be provided to adapt \\
to students' different levels of study achievement. \\
- Robot provides answers too directly and fast. \\
- Nine teachers failed to design a new scenario from \\
scratch. \\
- One teacher who majored in computer science can \\
design a new scenario from scratch. \\
- Many teachers want to try a new scenario that can \\
be a new template, but they said that the editor should be \\
easy to try. \\
- Regardless of design from scratch, the basic \\
template must be provided to avoid irritating and time \\
consuming jobs to build a new class scenario. \\
o Teachers usually use the example template with no \\
or little modification. \\
- Extraordinary teachers want to design new \\
templates and to share them with other teachers. \\
- Template should be categorized by the subjects, the \\
teaching method, and the curriculum \\
- Simulation in real time is too time consuming to \\
verify the class scenario. \\
- Simulation that is robot-centric is not proper. \\
- Simulation that is contents- and materials-centric is \\
more proper. \\
- Simulation is incapable of tracking the flow and \\
contents of a class. \\
- The number of example templates is insufficient to \\
refer to. \\
- Teachers want to share their class scenarios, \\
contents and materials with other teachers. \\
- Teachers do not want to sell their class scenarios, \\
contents and materials, but want to get some respect or \\
honor.
\end{tabular}

\section{Requirements Definition}

From the information collected by the interviews, we defined the requirements for the class scenario editor for teachers. We defined the requirements and drew a software mock-up to describe the requirements. By using the mock-up, we requested feedback from the teachers who had interviews and used this feedback to modify the requirements. After three iterations, the final requirements are defined as shown in Table 3.

From all subjects, we re-defined the user requirements for the class scenario editor. Legacy robot scenario editors and the class scenario editor have almost the same functions. However, the target user is completely different, so that the user requirements should also be re-defined. For example, most legacy users of robot simulators want to simulate the service scenario that is robot-centric. However, teachers have little interest in robots and want to verify their class scenario that is contents- and class materials-centric. 
Table 3. User Requirements

\begin{tabular}{|c|c|}
\hline Subject & Facts \\
\hline Terms & $\begin{array}{l}\text { - Terms in the editor should be general and easy } \\
\text { - Technical terms and engineering terms must not } \\
\text { be used } \\
\text { - Abbreviation should not be used } \\
\text { - Terms should be related with contents and } \\
\text { materials of class }\end{array}$ \\
\hline Navigation editor & $\begin{array}{l}\text { - Robot navigation editor should be edited from } \\
\text { templates } \\
\text { - Robot navigation editor should provide easy and } \\
\text { simple GUI } \\
\text { - Basic navigation should be designed to gather } \\
\text { attention of students before the start of class } \\
\text { - In the middle of class, navigation should be } \\
\text { minimized to help students keep focusing on class }\end{array}$ \\
\hline Motion editor & $\begin{array}{l}\text { - Motion editor should be edited from example } \\
\text { templates. } \\
\text { - Motion should be generated from the } 3 \mathrm{D} \text { motion } \\
\text { of user based on } 3 \mathrm{D} \text { sensors. } \\
\text { - Motion editor should provide easy and simple } \\
\text { GUI. } \\
\text { - Motion should not be fast and sudden to protect } \\
\text { students. }\end{array}$ \\
\hline Commander & $\begin{array}{l}\text { - User should be able to define commands using } \\
\text { STT and reuse commands. } \\
\text { - Commanding robots should not distract focusing } \\
\text { on teaching and learning. } \\
\text { - The accuracy and response time of voice } \\
\text { recognition for STT should be proper. } \\
\text { - Robots should recognize the voice of teachers. In } \\
\text { other words, robot should filter out student's undesired } \\
\text { command. }\end{array}$ \\
\hline Quiz & $\begin{array}{l}\text { - Robot should provide a proper level of quizzes to } \\
\text { the specific student. } \\
\text { - Robot should provide answers after enough time } \\
\text { and give some hints if students are struggling to find } \\
\text { the answer. }\end{array}$ \\
\hline Design from scratch & $\begin{array}{l}- \text { Designing a new scenario from scratch should be } \\
\text { an advanced function } \\
\bullet \text { Designing a new scenario from a template should } \\
\text { be a default function }\end{array}$ \\
\hline Template & $\begin{array}{l}\text { Various kinds of templates according to the } \\
\text { subjects, the teaching method, the curriculum and so } \\
\text { on should be provided. } \\
\text { - Examples of contents, multimedia files and class } \\
\text { materials for the template should be provided, along } \\
\text { with the template file. }\end{array}$ \\
\hline Simulator & $\begin{array}{l}\text { - Simulation should provide the function to control } \\
\text { the speed of simulation. } \\
\text { - Simulation should describe the robot's action, as } \\
\text { well as contents, multimedia and class materials so that }\end{array}$ \\
\hline
\end{tabular}




\begin{tabular}{|l|l|}
\hline Template sharing & all events are aligned to the time in the class. \\
- The class scenario editor should provide a \\
template sharing function. \\
- The template sharing function should have an \\
evaluating and ranking system to motivate the creators. \\
- Since some contents, multimedia and class \\
materials are too large to share directly by the editor, \\
the editor should support a cloud service to share large \\
files.
\end{tabular}

\section{Conclusion}

In South Korea, robotic systems have been deployed as teaching aids in elementary and middle schools to utilize the benefits of robot-aided classes. However, regardless of efforts in model schools over several years, teachers are still reluctant to use teaching assistant robots in school due to their difficulties in using the legacy robot scenario editor to design their own class scenario. To provide flexible and on-demand class scenarios, teachers should design and edit their own class scenarios to achieve their class objectives.

Since most teachers are usually not familiar with computer programming and robotics, a class scenario editor that is easy to use even without much expertise is required. We analyzed the requirements for developing a class scenario editor for robot-aided classes through interviews and experiments with teachers. Ordinary teachers do not want to develop a class scenario from scratch but rather want to modify and enhance the example templates. In addition, they want to simulate their class scenario with visual simulation. In addition, some extraordinary teachers who have expertise of computer programming and/or robotics want to design and provide example templates as a resource for other teachers. Therefore, the class scenario editor also has to offer a function for sharing the templates. From the user requirements, a class scenario editor for robot-aided classes can be developed and used by typical teachers without expertise about computer programming and robotics.

\section{Acknowledgements}

This work was supported by the National Research Foundation of Korea Grant funded by the Korean Government (NRF-2013S1A5A8020988).

\section{References}

[1] K.Y.Chin, Z.W. Hong and Y.L. Chen, "Impact of using an educational robot-based learning system on students' motivation in elementary education. Learning Technologies", IEEE Transactions on, vol. 7, no. 4, (2014), pp.333-345.

[2] H.S. Choi and K.H. Kim, "The Students' Perception Change in the Curriculum Implementation through the H.S.Robot-based Instruction", International Journal of Software Engineering and Its Applications, vol. 9, no. 6, (2015), pp.187-196.

[3] T. Han, and Y.H Seo, "Development of a Service Robot System for a Remote Child Monitoring Platform", International Journal of Smart Home, vol. 8, no. 5, (2014), pp.153-162.

[4] C.-Y. Chen and L.-H.Wang, "The motion editor and high precision integration for optimal control of robot manipulators in dynamic structural systems", Structural Engineering and Mechanics, vol. 41, issue 5, (2012), pp.633-644.

[5] Y. Kuroki, B. Blank, T. Mikami, P. Mayeux, A. Miyamoto, R. Playterand and J. I. Yamaguchi, "Motion creating system for a small biped entertainment robot. In Intelligent Robots and Systems", Proceedings. 2003 IEEE/RSJ International Conference on Intelligent Robots and Systems, vol. 2, (2003), pp. 13941399.

[6] D. Choi, J. Ha, M. Jung, M. Park and H. Park, "Development of Robot Scenario Script Language and Tool for Non-Expert", Journal of Automation and Control Engineering, vol. 3, no. 6, (2015), pp. 498502. 
[7] J. Peterson, G. D. Hager and P. Hudak, "A language for declarative robotic programming", In International Conference on Robotics and Automation, vol. 2, (1999), pp. 1144-1151.

[8] N. Bevan, "UsabilityNet methods for user centred design", Human-Computer Interaction: theory and Practice, vol. 1, (2003).

[9] C. Datta, J. Chandimal, I. Han Kuo and B. A. MacDonald, "RoboStudio: A visual programming environment for rapid authoring and customization of complex services on a personal service robot", In Intelligent Robots and Systems (IROS), 2012 IEEE/RSJ International Conference on, IEEE, (2012), pp. 2352-2357.

[10] Z.-W. Hong, Y.-M. Huang, M. Hsu and W.-W. Shen, “Authoring Robot-Assisted Instructional Materials for Improving Learning Performance and Motivation in EFL Classrooms", Journal of Educational Technology \& Society, vol. 19, no. 1, (2016).

[11] G. Kotonya and I. Sommerville, "Requirements Engineering: Processes and Techniques Chichester", UK: John Wiley and Sons, (1998)

[12] E. Lee and K. Lee, "Proposal of Class Scenario Design Method for Robot-aided Class", Advanced Science and Technology Letters, vol.122, no. IRTT 2016, (2016), pp.143-145.

[13] "HPI Racing", http://www.hpirobot.jp/.

[14] "ROBOTIS", http://support.robotis.com/en/.

[15] "NAO robot in Choregraphe", http://www.warp1337.com/content/simultaneous-nao-choregraphe-andblender-motion-control-xbox-kinect.

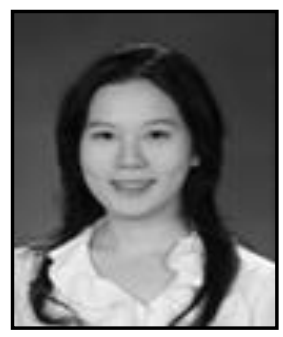

Eun-Mi Lee, she received her B.S. degree in fine art - painting from RMIT University, Melbourne, Australia, in 2003 and received her M.S. degree in art education from Hongik University, Seoul, Korea, in 2008. Since 2013, she has been a Ph.D student in the Department of Digital Media, Soongsil University. She is a committee member of Korea Fine Artist Association. Her current research interests include the areas of convergence of contemporary art and information technology, artificial intelligence system to understand abstract art and knowledge-based reasoning system. Contact her at vivienne@ssu.ac.kr.

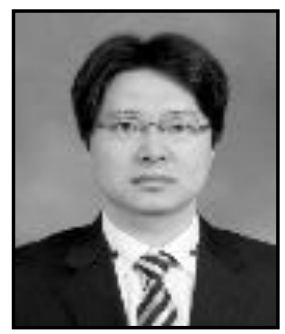

Kang-Hee Lee, he received his B.S., M.S., and Ph.D degrees in Electrical Engineering and Computer Science from Korea Advanced Institute of Science and Technology (KAIST), Daejeon, Korea, in 1999, 2001, and 2006, respectively. Since 2006, he has been a Senior Engineer in Digital Media \& Communication Research Center, Samsung Electronics Company, Ltd., Korea. $\mathrm{He}$ has been a dispatched researcher in the Robotics Institute, Carnegie Mellon University in 2008. Since moving to Soongsil University in 2009, he has been with Global School of Media, Soongsil University, Seoul, Korea. His current research interests include the areas of ubiquitous robotics, evolutionary robotics, emotional robotics, media robotics, cognitive task planning system, and knowledge-based reasoning system. Contact him at kanghee.lee@ ssu.ac.kr. 
International Journal of Control and Automation Vol. 9, No. 11 (2016) 